\title{
The first reported drug resistant pediatric HIV case in Sri Lanka
}

\author{
Ranatunga $\mathbf{J}^{1}$, Jayakody $\mathbf{C}^{1}$, Ranasinghe $\mathbf{S}^{1}$, Jagath $\mathbf{R}^{1}$

\section{Introduction}

HAART has reduced morbidity and mortality of HIV infected individuals worldwide. Antiretroviral therapy (ART) has given a new hope for people living with HIV. Universal access to first-line ART is becoming more of a reality in most low and middle income countries in Asia. However, second-line regimens are a challenge to most of the developing countries.

Even with ART, disease progression may still occur if effectiveness of the drug regimen fails over time due to many factors, the most encountered being adherence problems. Treatment failure manifests as an increase in viral load, decrease in CD4 count or appearance of clinical symptoms. The emergence of drug resistance is of increasing concern in countries where ART and ARV prophylaxis is widely used. It is a major issue in resorce limited settings where second and subsequent lines of therapy are not widely available.

\section{Case report}

One year and eight month old child with HIV infection found to have drug resistance during follow up.

The baby was born to a mother with retroviral infection, who was identified at 20 weeks of gestation in 2009 during a pilot project conducted in a leading maternity hospital in Sri Lanka. The universal screening for HIV in the antenatal group is not done in Sri Lanka yet. Mother's CD4 count was 800/ ul, ART prophylaxis with zidovudine mono therapy was given from 28 weeks of gestation according to the guidelines . The baby was delivered by Elective LSCS at 37 weeks of gestation. At delivery birth weight was $2.235 \mathrm{Kg}$ and baby had no postnatal complications. The baby was given sd NVP and AZT for seven days and was exclusively formula fed.

Child was given BCG vaccine one week after birth and was followed up. Co- trimoxazole prophylaxis was started. Baby's HIV antibody test was also positive which was done at 8 weeks of life.
Quantitative RNA PCR test was done at the age of 4 months due to unavailability of DNA PCR facilities and the viral load was $>750,000$ copies $/ \mathrm{ml}$. At this time, the child was hospitalized with severe diarrhoea and failure to thrive. At this stage, the child was symptomatic and in WHO stage 3. ART was started on 06/03/2010 with (D4T, 3TC, NVP) based regimen as the child was anemic. As there is no pediatric formulas of D4T, the child had to be given crushed combined adult pills of D4T $+3 \mathrm{TC}$. There were no facilities available for Therapeutic drug monitoring and the clinicians were not able to assess whether the child was maintaining adequate drug levels. With this regimen for 45 days child gained weight and Haemoglobin level was satisfactory.

Child developed BCG adenitis while on treatment was started on anti TB therapy. HB level was satisfactory at that time. Syrup ABC became available and the ART regimen was modified to AZT, 3TC, ABC regimen. While the child was on this regimen there were some social problems and child was not brought to the clinic for review and mother collected the medication and continued treatment without any follow up investigations.

Three months after initiating treatment, baby had severe anemia and the regimen was changed to 3TC, $\mathrm{D} 4 \mathrm{~T}, \mathrm{ABC}$ due to the unavailability of any other pediatric formulations in Sri Lanka.

Quantitative RNA PCR test was repeated at that time (after one year of starting ART) which revealed a viral load of 88209 copies/ml. This raised the possibility of drug resistance in this child. Even though the child had many difficulties with the unavailability of pediatric ART regimens, the drug adherence was optimal according to the mother. Viral resistance test was carried out with much effort with financial assistance from positive women's network, which revealed high level resistance to Nevirapine, Emptricitabine, lamivudine and possible resistance to abacavir and stavudine. Then the baby was switched

${ }^{1}$ National STD/AIDS Control Programme, Colombo, Sri Lanka. 
to a second line regimen which included ddI, ABC, LPV/RTV. This regimen was started with reference to the results of the resistance test and the availability of the formulations after many expert consultations. Some of the drugs were imported from India, with the assistance of donors.

Her CD\% is 47 after 4 months of therapy. Child is asymptomatic and attends Pre School also. A viral load test will be done after six months of being in the new regimen.

\section{Discussion}

Antiretroviral drugs (ARVs) are highly effective for the prevention of mother-to-child HIV transmission (PMTCT). Without ARV prophylaxis, the risk of transmission by 18 months of age ranges from 25$40 \%$ in breastfeeding populations. However, recent trials have demonstrated that several ARV regimens, administered to mothers and/or infants during pregnancy and breastfeeding, can reduce transmission to $1-8 \%$ at $6-12$ months of age (1). This case highlights a case of HIV infected child even after PMTCT interventions and the development of drug resistance. Possibilities of failure in PMTCT interventions in this case could be the result of high viraemia of mother during pregnancy. Although inexpensive and relatively easy to administer, sd NVP is less effective than currently recommended regimens which could also be a reason in this baby to be positive. There was evidence for Nevirapine resistance. That is a well known phenomenon with Nevirapine regimens given for PMTCT. It has been shown that transmission reduction is considerably more effective when combining sd NVP with two nucleoside reverse transcriptase inhibitors (NRTIs), such as zidovudine (AZT) and lamivudine (3TC). (2)

The emergence of drug resistance among HIV infected infants and children may result either from drug resistant strains being transmitted from the mother or a drug-resistant strain developing due to administration of paediatric ART or maternal or infant ARVs used for PMTCT or maternal ART. Development of HIV Drug Resistance in children on ART is usually related to poor adherence, use of suboptimal regimens, or to problems with drug absorption or pharmacokinetics. All of these factors give rise to sub therapeutic drug levels and the rebound of viraemia with resistant virus. (3)

Single dose NVP is prone to resistance formation and might impede subsequent treatment involving NVP or other NNRTIs while combining NVP with NRTIs has shown to reduce the emergence of NNRTI-resistant mutations (2). Possible cause of viral resistance in this case could be drug resistance following exposure to antiretroviral drugs during antenatal period and delivery or current ARV therapy. The baby was exposed to NVP during perinatal and post natal period as well as after initiating ART with NVP based regimen.

This case clearly shows the difficulties faced in managing pediatric HIV patients especially in resource poor countries.

Lack of sufficient pediatric formulations, lack of tests to confirm infection early and lack of ART trials in this age group is a problem experienced worldwide; a major burden born by developing countries.

\section{References}

1. Andrea L. Ciaranello, Freddy Perez, Matthews Maruva, Jennifer Chu, Barbara Engelsmann, Jo Keatinge, Rochelle P. Walensky, Angela Mushavi, Rumbidzai Mugwagwa, Francois Dabis, Kenneth A. Freedberg. 2010 Guidelines for Prevention of Mother-to-Child HIV Transmission in Zimbabwe: Modeling Clinical Outcomes in Infants and Mothers. PLoS One [serial online] 2011 June [cited 2012 Aug 03]; 6(6): e20224. Available from: URL:http://www.ncbi.nlm.nih.gov

2. Inga Kirsten, Julius Sewangi, Andrea Kunz, Festo Dugange, Judith Ziske, Brigitte Jordan-Harder, Gundel Harms, Stefanie Theuring. Adherence to Combination Prophylaxis for Prevention of Mother-to-Child-Transmission of HIV in Tanzania. PLoS One [serial online] 2011 June [cited 2012 Jul 23]; 6(6): e21020. Available from: URL:http://www.ncbi.nlm.nih.gov

3. Antiretroviral therapy for HIV infection in infants and children: towards universal access: recommendations for a public health approach. Switzerland, Geneva: World Health Organization; 2010. P.83 